\title{
Supplement: Super-cooled liquid fogs over the central Greenland ice sheet
}

Christopher J. Cox ${ }^{12}$, David C. Noone ${ }^{3}$, Max Berkelhammer ${ }^{4}$, Matthew D. Shupe ${ }^{12}$, William D. Neff ${ }^{12}$, Nathaniel B. Miller', Von P. Walden', Konrad Steffen ${ }^{6}$

5 Cooperative Institute for Research in Environmental Sciences, Boulder, Colorado, 80309, USA

2 NOAA Earth System Research Laboratory, Boulder, Colorado, 80305, USA

${ }^{3}$ College of Earth, Ocean, and Atmospheric Sciences, Oregon State University, Corvallis, Oregon, 97331, USA

Department of Earth and Environmental Sciences, University of Illinois at Chicago, Chicago, Illinois, 60607, USA

s Department of Civil and Environmental Engineering, Washington State University, Pullman, Washington, 99164, USA

10 'Swiss Federal Research Institute WSL, Birmensdorf, CH-8903, Switzerland

Correspondence to: Christopher J. Cox (christopher.j.cox@noaa.gov)

This supplemental information describes the details of the processing of the DMT Fog Monitor 100 (FM100s)

15 principally based on the recommendations Spiegel et al. (2012), as described in Section 2 of the main text.

\section{Mie Scattering Ambiguity}

The non-monotonic nature of the Mie scattering function causes sizing ambiguities, which are well documented (Spiegel et al., 2012 and references therein). These ambiguities arise because the same scattering cross section may apply to multiple particle sizes, resulting in spurious modes in the distributions of sampled particles (Baumgardner et al., 2010).

Figure $\mathrm{S} 1$ shows the Mie scattering functions calculated for liquid spheres $(\mathrm{n}=1.33+0 \mathrm{i}, \lambda=658 \mathrm{~nm})$. The scattering cross sections are calculated for the configuration of the instrument optics by scaling the particle cross section to the normalized intensity integrated over the angles subtended by the path to the detector. These angles are not precisely known and also depend on the location of the particle in the instrument transmission path, so upper and lower bounds are estimated by performing a series of calculations for a range of angular pathways between $3^{\circ}$ and $12.6^{\circ}$. More details about the instrument optical configuration and the Mie calculations can be found in Spiegel et al. (2012). The grey boxes in the figure show the 20 default particle size bins. To reduce the influence of sizing ambiguities, previous studies have suggested 
reconfiguring the bin limits (Gonser et al., 2011; Spiegel et al. 2012), combining bins (Pinnick and Auvermann, 1979; Dye and Baumgardner, 1984), smoothing distributions (Gonser et al., 2011), and more sophisticated statistical methods (Spiegel et al. 2012). For the Summit field program, the default bin limits were used; a reconfiguration of the bins is only possible prior to deployment. Gonser et al. (2011) proposes smoothing the PDFs with a Savitsky-Golay filter, but we find that the smoothed PDF can be sensitive to the parameter choices in the convolution filter and therefore we do not smooth the distributions. We instead rely on combining ambiguous bins, as described in the main text. Figure S1 indicates a large amount of ambiguity between 4 and $10 \mu \mathrm{m}$, which corresponded to a persistent mode in the raw observations between 6 and $8 \mu \mathrm{m}$. We therefore combined the three default bins between 4 and $10 \mu \mathrm{m}$. A similar, but less influential problem occurs near $23 \mu \mathrm{m}$, but no consolidation of these bins is applied.

The temperature at Summit is nearly always below $0{ }^{\circ} \mathrm{C}$. Therefore, the particles sampled by the FM100 are typically composed of ice or super-cooled liquid. In all cases, the particle size should be interpreted as a diameter for an optically equivalent sphere in the Mie regime. For ice, which is non-spherical, this metric is typically smaller than geometric definitions for ice size (Borrmann et al., 2000). An index of refraction of 1.33+0i (liquid, $658 \mathrm{~nm}$ ) is assumed by the software for determining particle size. The oscillations in the scattering function for a refraction of $1.31+0 \mathrm{i}$ (ice, $658 \mathrm{~nm}$ ) is shifted slightly in phase from that of liquid (see also Baumgardner, 1992; Gayet et al., 1996), but this does not substantially increase uncertainty (Figure S1).

\section{Sampling Losses}

Spiegel et al. (2012) also characterized the sampling losses in the FM100 that arise from actively drawing air samples through the instrument following previous work that calculated analogous sampling efficiencies for aerosol scattering probes (Liu and Aragwal, 1974; Hinds, 1982; von der Weiden et al., 2009; Brockman, 2011). The efficiency calculations fall into two main categories. The first is aspiration efficiency, which is the proportion of particles in the ambient air that are drawn into the instrument. The second is transmission efficiency, which is the proportion of aspirated particles that pass through the instrument. Losses in transmission occur for five reasons; gravitational settling in the wind tunnel and the contraction horn, turbulent deposition in the wind tunnel and contraction horn, and inertial losses in the contraction horn (Spiegel et al., 2012). 
These losses are functions of particle size, wind velocity, and wind direction with respect to the inlet horn $\left(\theta\right.$, where $\theta=0^{\circ}$ is defined as directly into the inlet horn). The equations are valid for $|\theta|<90^{\circ}$. Therefore, when the wind is oriented in the same direction as the instrument (i.e., $|\theta|>90^{\circ}$, northerly winds at Summit), the sampling losses are unknown, but expected to be large.

Figure S2 shows the results of the calculations in a manner similar to Spiegel et al. (2012). In this work, the calculations are used for two purposes: 1) to determine the limits of the wind directions suitable for analysis $\left(|\theta|<50^{\circ}\right.$ is used for the analysis for Figs. 8-11 in the main text), and 2) to correct the number concentrations for each size bin for those directions. In general, the sampling efficiencies are lower for larger particles and larger $|\theta|$ angles. The sampling efficiency calculations were derived assuming spherical particles (Figs. S2a and S2b). Particles in super-cooled-liquid fogs can be

10 assumed to have spherical habit and spherical habit may be a close approximation for ice fogs, which are typically droxtals at the lower end of the size spectrum (Schmidt et al., 2013 and references therein). Samples composed of diamond dust, precipitation and blowing snow likely exhibit a variety of habits, some with large aspect ratios. Thus, these calculations may not be a good representation for the non-spherical samples. Ice is also lower in density than liquid by about $9 \%$. We recalculated the results for spherical particles with ice density (Fig. S2c), indicating a slightly conservative estimate of

15 particle losses, but otherwise, relatively little sensitivity to assuming liquid density. To estimate the effect of habit, we calculated sampling efficiencies for ice density using cylinders oriented with the long axis perpendicular to the direction of flow (Hinds 1999). The results (Fig. S2d) indicated that the sampling efficiency is slightly higher than for spherical particles. Taken together, misrepresenting density and habit in calculating sampling efficiency of ice by assuming liquid spheres is expected to produce a conservative estimate of sampling efficiency (thus, an overestimation of losses).

\section{References}

Baumgardner D., Kok G., and Chen P.: Multimodal size distributions in fog: Cloud microphysics or measurement artefact? 5th International Conference on Fog, Fog Collection and Dew, Münster, Germany, 25 July-30 July 2010. 146, 2010.

Baumgardner D., Dye J. E., Gandrud B. W., Kollenberg R. G.: Interpretation of measurements made by the Forward Scattering Spectrometer Probe (FSSP-300) during the Airborne Arctic Stratospheric Expedition, J. Geophys. Res., 97, 8035-

25 8046, doi: 10.1029/91JD02728, 1992. 
Borrmann, S., Luo, B., and Mishchenki, M.: Application of the T-Matrix method to the measurement of aspherical (ellipsoidal) particles with forward scatting optical particle counters, J. Aerosol Sci., 31, 789-799, doi:10.1016/S00218502(99)00563-7, 2000.

5

Brockmann, J. E.: Aerosol Transport in Sampling Lines and Inlets. In Aerosol Measurement: Principles, Techniques, and Applications, Kulkarni, P, Baron, P, and Willeke, K (eds). John Wiley \& Sons: 69-105, doi: 10.1002/9781118001684.ch6, 2011.

Dye, J. E., and Baumgardner, D.: Evaluation of the forward scattering spectrometer probe, I: Electronic and optical studies, 10 J. Atmos. Ocean Tech., 1, 329-344, doi:10.1175/1520-0426(1984)001<0329:EOTFSS>2.0.CO;2, 1984.

Gayet J.-F., Febvre G., and Larsen H.: The reliability of the PMS FSSP in the presence of small ice crystals, J. Atmos. Ocean. Tech., 13, 1300-1310, doi: 10.1175/1520-0426(1996)013<1300:TROTPF>2.0.CO;2, 1996.

15 Gonser S. G., Klemm O., Griessbaum F., Chang S.-C., Chu H.-S., Hsia Y.-J.: The relation between humidity and liquid water content in fog: An experimental approach. Pure Appl. Geophys., 169, 821-833, doi:10.1007/s00024-011-0270- x, 2011.

Hinds, W. C.: Aerosol Technology: Properties, Behavior and Measurement of Airborne Particles. John Wiley and Sons: New York; 424 pp, 1982.

Liu B. and Agarwal J.: Experimental observation of aerosol deposition in turbulent flow, J. Aerosol Sci., 5, 145-155, doi: 10.1016/0021-8502(74)90046-9, 1974.

Pinnick, R. and Auvermann, H.: Response characteristics of Knollenberg light-scattering aerosol counters, J. Aerosol Sci., 10, 55-74, doi:10.1016/0021-8502(79)90136-8, 1979.

Spiegel, J. K., Zieger, P., Bukowiecki, N., Hammer, E., Weingartner, E., and Eugster, W.: Evaluating the capabilities and 25 uncertainties of droplet measurements for the fog droplet spectrometer (FM-100), Atmos. Meas. Tech., 5, 2237-2260, doi:10.5194/amt-5-2237-2012, 2012.

von der Weiden S.-L., Drewnick F., Borrmann S.: Particle Loss Calculator - a new software tool for the assessment of the performance of aerosol inlet systems, Atmos. Meas. Tech., 2, 479-494, doi:10.5194/amt-2-479-2009, 2009. 


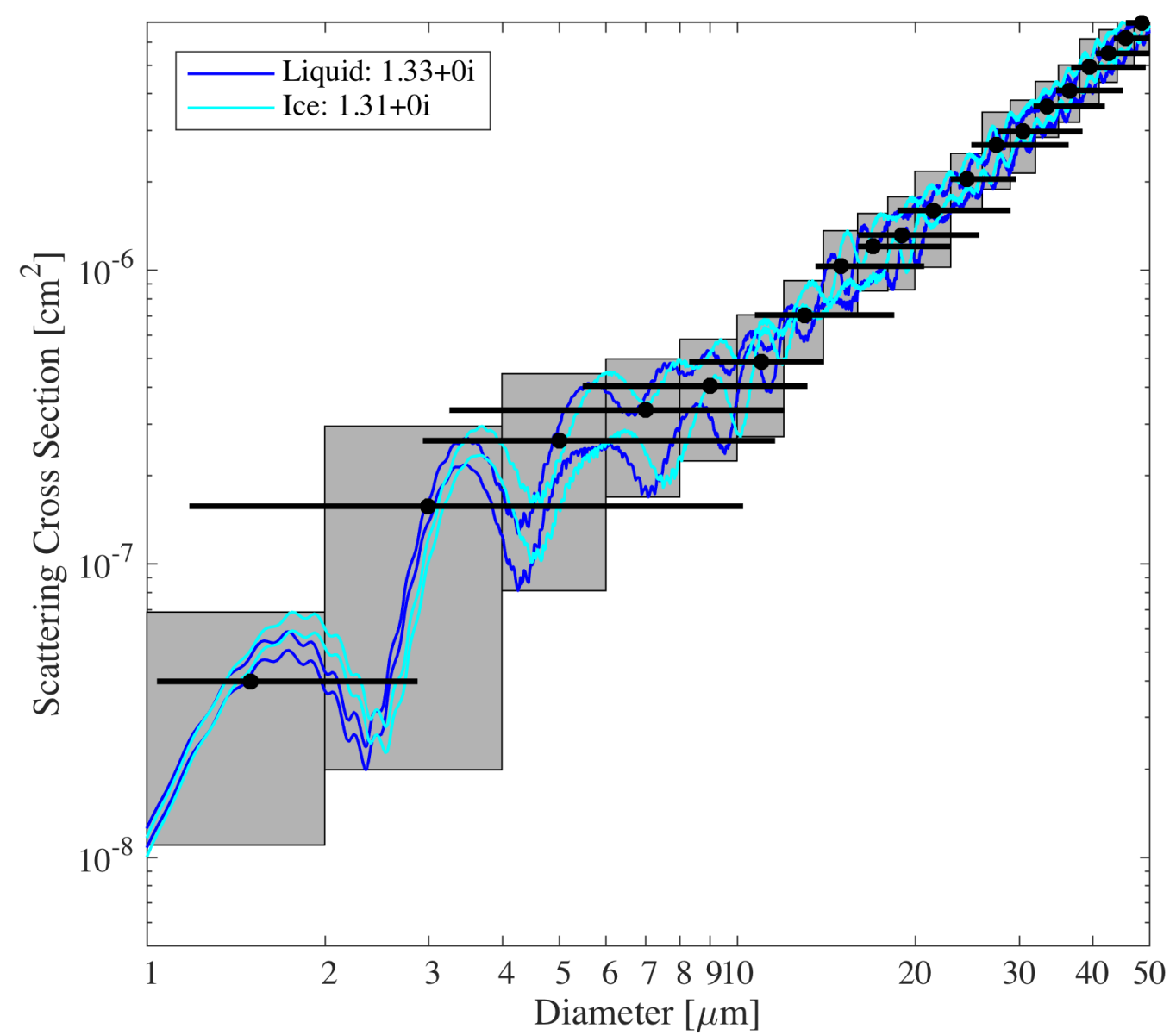

5

Figure S1. Theoretical Mie scattering cross sections for liquid and ice spheres, as would be measured at the detector of the FM100. Upper and lower bounds represent uncertainty in the optical set up. Grey boxes represent the original bin limits used by the FM100 software. Horizontal black lines represent uncertainty in the bin size assigned to a particle due to the nonmonotonic function (i.e., most scattering cross sections can be related to multiple sizes). The uncertainty includes both the 10 ice and liquid calculations. 
a)
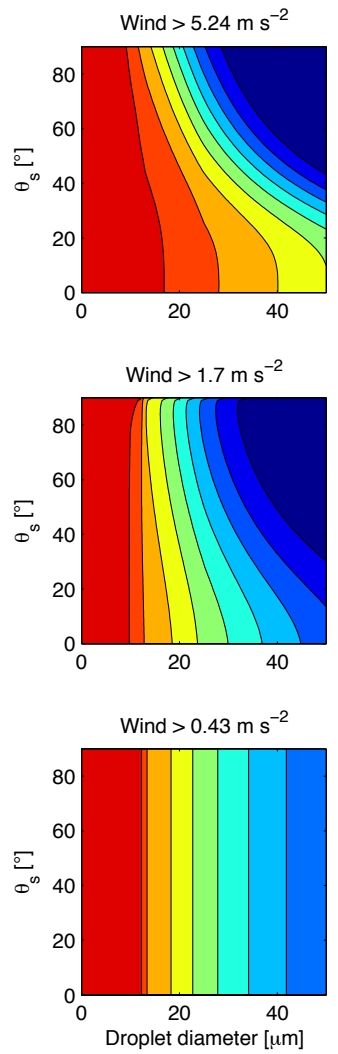

b)
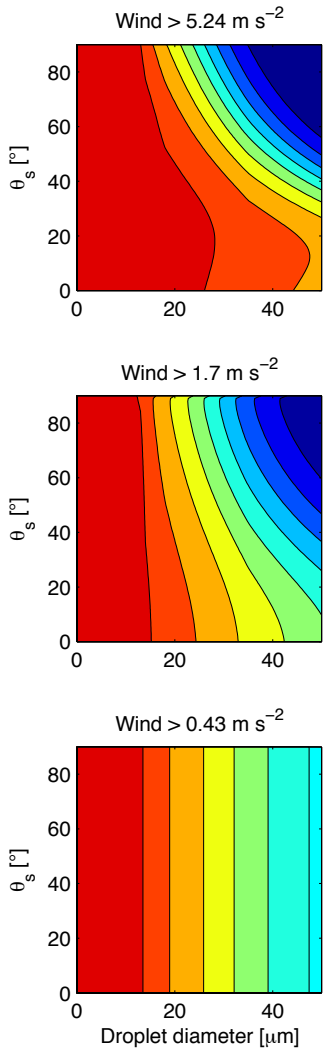

c)
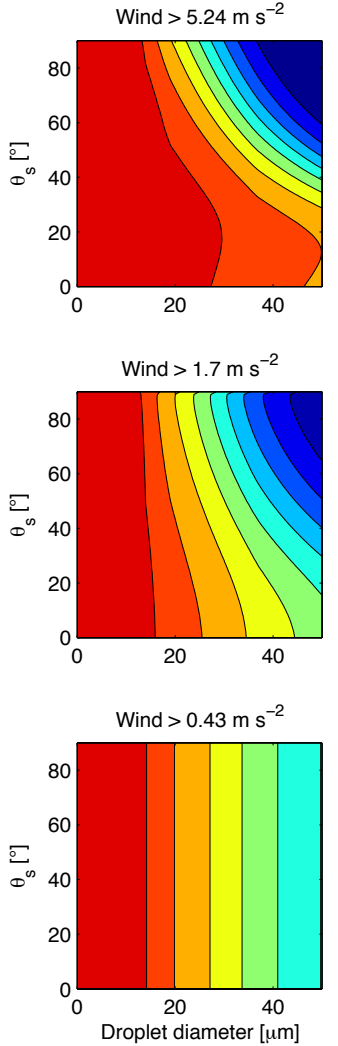

d)
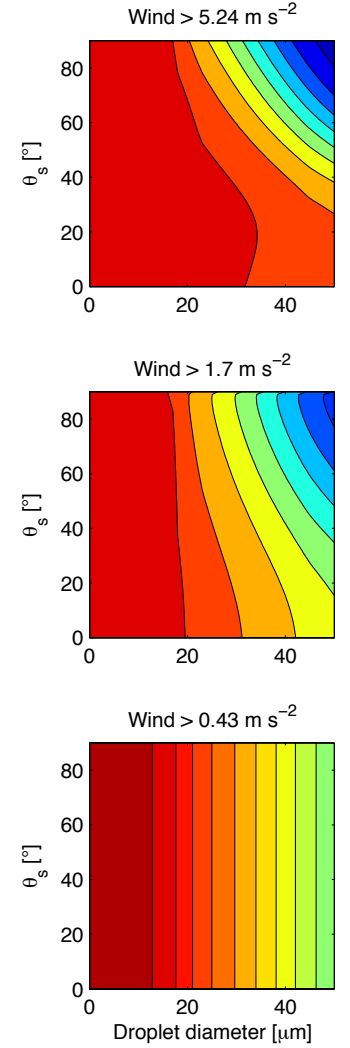

Figure S2. Calculations of sampling efficiency (fractional, colours) as a function of droplet diameter (x-axis) and sampling

5 angle, $\theta$, (y-axis). Rows represent different wind velocity conditions. (a) intake velocity $15 \mathrm{~m} \mathrm{~s}$ (liquid spheres), (b) intake velocity $7.5 \mathrm{~m} \mathrm{~s}^{-1}$ (liquid spheres) and (c) ice spheres $\left.\left(7.5 \mathrm{~m} \mathrm{~s}^{-1}\right), \mathrm{d}\right)$ ice cylinders $\left(7.5 \mathrm{~m} \mathrm{~s}^{-1}\right)$. 